\title{
No Support for Gravitational Waves
}

MUCH can be learnt about the methods of science from situations in which discoveries of a rather contentious nature are made by one scientist or group, and are then searched for by other interested workers, often without success. During the past year or two there seems to have been a rash of examples. In 1968, cosmic ray physicists at the University of Utah announced that a rather well established behavioural pattern of the intensity of cosmic ray muons with zenith angle had been found to break down at energies above several hundred GeV (Phys. Rev. Lett., 19, 1487; 1967). Their results seemed entirely convincing, yet nobody else has been able to reproduce them, either with similar experiments or by approaching the problem from a different standpoint. Professor C. B. McCusker's triumphal announcement to the last international cosmic ray conference that he had found the elusive quark hiding in the cores of extensive air showers has likewise received no corroboration (Phys. Rev. Lett., 23, 658; 1969). The latest candidate for addition to the list of unsubstantiated discoveries is gravitational radiation-but the problems entailed in proving or disproving it are immense.

In 1969 Professor J. Weber reported that he had detected gravitational waves (Phys. Rev. Lett., 22, $1320 ; 1969)$. This was the culmination of years of patient work on two rather carefully designed detectors situated 1,000 $\mathrm{km}$ apart at the Argonne National Laboratory and the University of Maryland. The vibrations of two large metal cylinders with natural frequencies about $1,660 \mathrm{~Hz}$ are measured by piezoelectric instruments and coincidences between the two signals are taken to indicate the existence of gravitational waves. But where is the supporting evidence? Weber continues to find coincidences between his detectors and has even recently suggested that the greater part of the gravitational radiation he detects comes from the galactic centre.

In this issue (page 346), thirteen workers from five laboratories in Great Britain report attempts to confirm Weber's findings by searching for the small amount of radio frequency energy almost certainly accompanying gravitational waves. At each of five sites (Cambridge, Dublin, Glasgow, Harwell and Jodrell Bank), a pair of dipole aerials was set up to detect radiation arriving within a frequency band of $1 \mathrm{MHz}$ about $151 \mathrm{MHz}$ from almost anywhere in the sky. A number of coincidences between two of the stations were found, but tests have shown that it is unlikely that they are attributable to gravitational waves. Five coincidences were observed between three of the stations, but none of these was consistent with emission from a distant source. No coincidences, however, have been observed between four or all five of the stations. The outputs of the stations have also been examined. Since Weber's suggestion that the galactic centre may be a source of gravitational waves, the collaborators in the radio waves have put a direc- tional detector in Malta and have replaced four of the original five detectors with directional types. It is possible with this array to observe the galactic centre and look for radio pulses which could be compared in time with the coincidences observed by Weber with his own apparatus. This could, however, pose difficulties because of the separation of the two experiments on the Earth's surface and the small probability of the two sets of apparatus observing the galactic centre at the same time.

Negative results from their experiment do not by any means invalidate Weber's original claims. There could be many reasons why radio pulses associated with gravitational waves may not be observable. The radio waves could be absorbed between their source and the Earth or, indeed, there may be no radio emission associated with gravitational waves. This, however, seems unlikely, for the violent conditions necessary to emit the enormous amounts of energy in the form of gravitational waves are almost certain to provide also the ideal source for radio signals.

\section{LIQUIDS \\ Electrical Switching in Alloys}

by our Solid State Physics Correspondent

The disclosure by G. Busch, H. J. Guentherodt, H. U. Kuenzi and A. Schweiger (Phys. Lett., 33, 64; 1970) that a liquid alloy of tellurium and selenium has the electrical properties of a switch comes very much as a bonus in the search for a non-crystalline version of this important circuit element. So far little headway has been made in explaining the switching behaviour in amorphous solids discovered by Ovshinsky two years ago, and liquids are always one degree harder to understand. But the common features in the disordered structures of liquids and amorphous solids offer the chance to pick out some fresh clues as to why the electrical resistance of these materials should dive so sharply on a small change of voltage, and may help to point to the type of amorphous substance which can perform this function most efficiently.

Busch et al. found a sharp transition between resistive and conductive state in various alloys of both tellurium and selenium and tellurium and sulphur. They observed switching behaviour up to $200^{\circ} \mathrm{C}$ above the melting point with the samples held between graphite or platinum electrodes. The property was apparent from the sharp breaks in the current voltage characteristic.

In the simplest form of switch the resistance changes reversibly as the voltage is altered. It is, however, possible to obtain bistable switching action in which the device remains indefinitely in the desired "on" or "off" state until specifically switched out of it. The understanding of solid state switching is still too insecure for anybody to be sure whether the latter type of memory switch is related to the former variety or if it arises from an essentially different electronic process. 